\title{
COMPENSAÇÃO DE DESEQUILÍBRIOS DE CARGA EMPREGANDO CONVERSOR ESTÁTICO OPERANDO COM MODULAÇÃO EM LARGURA DE PULSO
}

\author{
RODRIGO CUTRI ${ }^{*} * *$ E LOURENÇO MATAKAS JUNIOR** \\ ** EPUSP - Departamento de Energia e Automação - PEA- Universidade de São Paulo - USP \\ Av. Prof. Luciano Gualderto , trav.3 ,n ${ }^{\circ} 158$ - 05508-900 - São Paulo,SP - BRASIL - \\ Tel :+55(11)3091-5483 \\ e-mail : matakas@pea.usp.br \\ * IMT - Instituto Mauá de Tecnologia - Escola de Engenharia Mauá - E.E.Mauá \\ Departamento de Engenharia Elétrica - DEL \\ Praça Mauá ,nº1 - 09580-900 - São Caetano do Sul, SP - BRASIL - Tel:+55(11)4239-3000 \\ e-mail : rodrigo.cutri@maua.br
}

\begin{abstract}
Resumo -É cada vez maior a preocupação com a qualidade da energia elétrica tanto do ponto de vista do consumidor quanto da concessionária. Um dos grandes problemas encontrados é o desequilíbrio de correntes trifásicas devido à utilização de cargas monofásicas, bifásicas ou trifásicas desequilibradas em sistemas trifásicos de potência. Para eliminar esse desequilíbrio é necessária a identificação da parcela de corrente de seqüência negativa presente no sistema e sua eliminação. A injeção das correntes de compensação via conversores estáticos operando em PWM (Pulse Width Modulation) é uma das soluções aplicadas. Esta solução exige o cálculo em tempo real da componente instantânea de seqüência negativa, que será utilizada para a obtenção da referência de corrente do conversor. Neste trabalho, um novo método é proposto para a extração em tempo real da componente da corrente de seqüência negativa, apresentando um bom desempenho em comparação com os outros métodos existentes. O desempenho de um compensador completo é verificado via simulação numérica, utilizando o software MATLAB (SimulinkPower System Blockset), através de um conversor do tipo fonte de tensão operando em PWM, incluindo as malhas de controle de corrente do lado $\mathrm{CA}$ e da tensão no barramento $\mathrm{CC}$.
\end{abstract}

Palavras-Chave - Cargas desbalanceadas, Detecção de seqüência negativa, Filtros Ativos

\section{COMPENSATION OF UNBALANCED LOADS WITH STATIC CONVERTERS USING PULSE WIDTH MODULATION}

Abstract - It's large the concern with the power quality, not only from the consumer's point of view but also from the utilities point of view. One of the most common problems is the unbalanced three-phase

Artigo Submetido em 22/03/2004. Primeira Revisão em 20/05/2004. Segunda Revisão em 04/07/2004. Aceito sob recomendação do Editor Geral Prof. Carlos Alberto Canesin. currents due to the use of single-phase loads, two-phase loads or unbalanced three-phase loads in three-phase power systems. To eliminate this unbalance it is necessary to identify the negative sequence components of the load current and to eliminate it. The injection of the compensating currents by means of Static converters using PWM (Pulse Width Modulation) is one possible solution. This solution needs an algorithm for the real time extraction of the negative sequence component that will be used for generating the reference current for the converter. In this work, a new method, faster than the existing well known methods, is proposed. It is fully simulated, with Matlab Simulink Power System Blockset, using a voltage source converter operating with PWM.

Keywords - Active Filter, Negative sequence, Unbalanced loads.

\section{INTRODUÇÃO}

Distorções e desequilíbrios nas correntes que circulam pela rede elétrica provocam diversos efeitos negativos. Equipamentos eletrônicos alimentados por retificadores (aplicações em eletrodomésticos, drivers de motores, reatores eletrônicos para iluminação, fornos de indução etc.), por exemplo, têm comportamento não linear, drenando correntes distorcidas da rede elétrica, mesmo quando alimentados por tensão perfeitamente senoidal. Os efeitos negativos destes harmônicos de corrente são notados no sobreaquecimento de dispositivos elétricos (máquinas rotativas, transformadores e capacitores de correção de fator de potência) e distorções na forma senoidal da tensão de fornecimento, o que pode prejudicar o funcionamento de circuitos mais sensíveis. Sistemas desbalanceados causam efeitos indesejáveis como perdas adicionais em motores e geradores com evidente redução na vida útil desses equipamentos, atuação da proteção contra sobrecarga provocada pela circulação de correntes de seqüência negativa em motores de indução, aumento da ondulação na tensão de saída dos retificadores e saturação de transformadores [28], [29], [31], [32] e [34].

Dentre as soluções para a compensação do desequilíbrio de cargas destacam-se o uso de transformadores para mudança do número de fases [4], [9] e [13]; a inserção de 
impedâncias [3], [12], [20], [21], [27], [28], [30] e [36], e a injeção de correntes via conversor estático operando em PWM [1], [3], [8], [10], [14], [15], [18], [19], [21], [25], [31], [33], [34] e [37]. Esta última solução é o foco deste artigo.

A injeção de correntes via conversor estático operando em PWM exige o cálculo em tempo real do componente instantâneo de seqüência negativa, que será utilizado para a obtenção da referência de corrente do conversor. Várias estratégias foram desenvolvidas para a compensação de desequilíbrios de cargas utilizando esta solução, dentre elas destacam-se: o sistema de referência síncrona positiva (RSP) e negativa (RSN) [10] e [31], a detecção em tempo real através de vetores espaciais (DTRVE) [37], o método da teoria de potência ativa e reativa instantânea (PQ) [2] e a injeção direta de corrente de seqüência negativa (DSNI) [11]. Uma explanação mais detalhada sobre os principais métodos é apresentada em [38]. Uma comparação entre algumas destas estratégias é vista em [7], [11] e [23].

Neste artigo, um novo método [11] baseado na teoria de componentes simétricas sem nenhuma transformação de base, utilizando apenas cálculos algébricos é proposto (item III). Uma comparação [38] entre as principais estratégias já conhecidas (RSP, RSN, DTRVE, PQ) é apresentada (item IV). A implementação do conversor de injeção de corrente de compensação é sucintamente apresentada no item V. Utilizando-se o software MATLAB (Simulink - Power System Blockset), o método proposto de extração é totalmente simulado (item VI) utilizando um conversor do tipo fonte de tensão operando em PWM, incluindo as malhas de controle da corrente CA e da tensão no barramento CC.

\section{INJEÇÃO DE CORRENTES VIA CONVERSOR OPERANDO EM PWM}

Uma solução para a compensação de desequilíbrios é a injeção de correntes de seqüência negativa através de conversores autocomutados do tipo PWM. O diagrama em blocos do sistema de compensação ativa utilizando um conversor comutado do tipo PWM é apresentado na Fig.1.

As correntes da carga $\left(\mathrm{i}_{\mathrm{r}}(\mathrm{t}), \mathrm{i}_{\mathrm{s}}(\mathrm{t}), \mathrm{i}_{\mathrm{t}}(\mathrm{t})\right)$ são medidas e um algoritmo efetua o cálculo das correntes de referência $\left(i_{\text {ref }} r\right.$-, $\left.\mathrm{i}_{\text {ref_s- }}, \mathrm{i}_{\text {ref } \mathrm{t}-}\right)$ a serem injetadas pelo conversor $\left(\mathrm{i}_{\mathrm{r}-}(\mathrm{t}), \mathrm{i}_{\mathrm{s}-\mathrm{t}}(\mathrm{t}), \mathrm{i}_{\mathrm{t}-}\right.$ $(\mathrm{t}))^{-}$, de modo que as correntes resultantes na rede $\left(\mathrm{i}_{\mathrm{rc}}(\mathrm{t}), \mathrm{i}_{\mathrm{sc}}(\mathrm{t}), \mathrm{i}_{\mathrm{tc}}(\mathrm{t})\right)$ sejam balanceadas. As correntes injetadas $\left(\mathrm{i}_{\mathrm{r}-}\right.$ $\left.(t), i_{s}(t), i_{t}(t)\right)$ são controladas a fim de se garantir que sigam com a maior fidelidade possível os valores de referência calculados ( $\left.\mathrm{i}_{\text {ref_r- }}, \mathrm{i}_{\text {ref_s- }}, \mathrm{i}_{\text {ref_t- }}\right)$.

Esta estratégia possui as seguintes características:

- menor volume, menor peso e maior custo quando comparada ao método de inserção de impedâncias;

- $\quad$ rápida compensação para cargas variáveis;

- pequena injeção de harmônicos e possibilidade de compensação dos harmônicos da corrente da carga;

- a potência ativa consumida pelo conversor resumese à necessária para suprir as perdas no conversor;
- possibilidade de escolha de compensação total (potência reativa, harmônicos e desequilíbrios) ou compensação parcial, incluindo qualquer combinação de desequilíbrio, reativos e harmônicos [26][34].

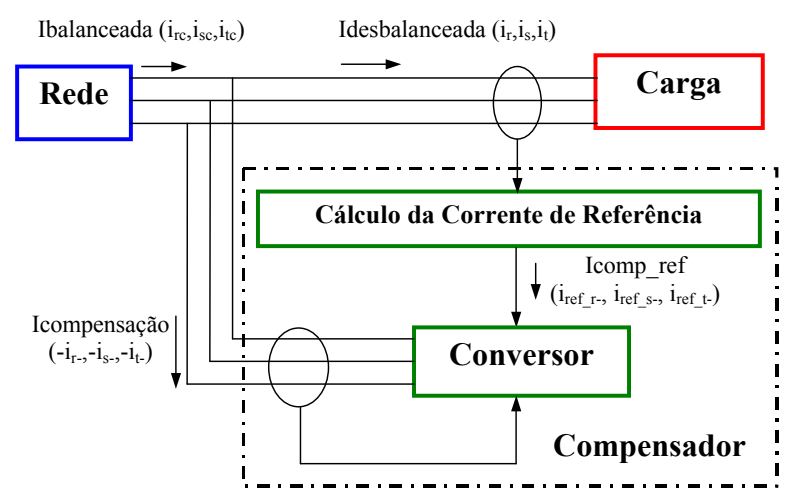

Fig. 1. Diagrama em blocos do compensador ativo de desequilíbrios

Usualmente, o valor da potência aparente associada aos reativos e desequilíbrios é maior do que a associada aos harmônicos. Assim, a compensação de distúrbios de baixa freqüência (reativos e desequilíbrios) é feita por um conversor chaveado de elevada potência operando com menor freqüência de chaveamento enquanto que os harmônicos podem ser tratados com um conversor de menor potência e alta freqüência de chaveamento.

\section{MÉTODO PROPOSTO-INJEÇÃO DIRETA DE SEQÜÊNCIA NEGATIVA (DSNI)}

A utilização do método para a obtenção das correntes instantâneas de compensação para um conversor do tipo PWM-VSI é proposta [11], o qual é baseado na teoria de componentes simétricos [29] e [39].

$\mathrm{Na}$ determinação desta solução, os harmônicos foram negligenciados. A eliminação das correntes de seqüência negativa é feita sem nenhuma transformação de sistema de coordenadas, operando diretamente com as correntes de linha medidas.

As correntes instantâneas de seqüência negativa necessárias a compensação $\left(\mathrm{i}_{\mathrm{r}-}(\mathrm{t}), \mathrm{i}_{\mathrm{s}-}(\mathrm{t}), \mathrm{i}_{\mathrm{t}-}(\mathrm{t})\right)$ podem ser calculadas diretamente a partir dos valores instantâneos das correntes de linha da carga $\left(\mathrm{i}_{\mathrm{r}}(\mathrm{t}), \mathrm{i}_{\mathrm{s}}(\mathrm{t}), \mathrm{i}_{\mathrm{t}}(\mathrm{t})\right)$ e dos valores instantâneos das correntes de linha da carga atrasados de $1 / 4$ de ciclo da fundamental $\left(\mathrm{i}_{\mathrm{r} 90}(\mathrm{t}), \mathrm{i}_{\mathrm{s} 90}(\mathrm{t}), \mathrm{i}_{\mathrm{t} 90}(\mathrm{t})\right)$ através da eq.(1), cuja dedução se encontra no anexo $\mathrm{A}$.

$$
-\left[\begin{array}{l}
i_{r-}(t) \\
i_{s-}(t) \\
i_{t-}(t)
\end{array}\right]=-\frac{1}{3} \cdot\left[-\left[\begin{array}{ccc}
-1 & 1 / 2 & 1 / 2 \\
1 / 2 & -1 & 1 / 2 \\
1 / 2 & 1 / 2 & -1
\end{array}\right] \cdot\left[\begin{array}{l}
i_{r}(t) \\
i_{s}(t) \\
i_{t}(t)
\end{array}\right]+\left[\begin{array}{ccc}
0 & \frac{\sqrt{3}}{2} & -\frac{\sqrt{3}}{2} \\
-\frac{\sqrt{3}}{2} & 0 & \frac{\sqrt{3}}{2} \\
\frac{\sqrt{3}}{2} & -\frac{\sqrt{3}}{2} & 0
\end{array}\right] \cdot\left[\begin{array}{l}
i_{r 90}(t) \\
i_{s 90}(t) \\
i_{t 90}(t)
\end{array}\right]\right]
$$


Este atraso é facilmente implementado armazenando-se as $\mathrm{N} / 4$ últimas amostras medidas onde $\mathrm{N}$ é igual ao número de amostras por ciclo da rede.

\section{ANÁLISE COMPARATIVA DOS MÉTODOS DE EXTRAÇÃO DA CORRENTE DE SEQÜÊNCIA NEGATIVA}

Em [38], os diversos métodos (DTRVE, PQ, RSP, RSN e DSNI) foram simuladas no software MATLAB - Simulink (Power Systems Blockset) modelando-se o inversor de potência como uma fonte de corrente ideal controlada e utilizando-se uma carga variável conforme descrito no item V. Deste modo, desprezam-se os efeitos do chaveamento, concentrando-se no comportamento em baixa freqüência, obtendo-se assim uma simulação mais eficiente do ponto de vista do tempo de processamento e da quantidade de pontos armazenados.

Todas as simulações foram efetuadas com condições iniciais quiescentes. Foram utilizadas tensões de fase-neutro simétricas e equilibradas com amplitude de $1 \mathrm{~V}$ e freqüência de $60 \mathrm{~Hz}$. Os métodos foram comparados obtendo-se as seguintes conclusões :

- o tempo de resposta aproximado em ciclos para os diversos métodos foi de 1 ciclo (DTRVE) , 2 ciclos (PQ, RSP, RSN) e $1 / 4$ de ciclo (DSNI);

- o grau de desequilíbrio obtido foi menor de : $1.5 \%$ para o método DTRVE (utilizando um filtro passa baixa de $4^{\circ}$ ordem, $\left.100 \mathrm{~Hz}\right) ; 1 \%, 2 \%$ e $1.5 \%$ respectivamente para os métodos PQ, RSP, RSN (utilizando um filtro passa baixa de $2^{\circ}$ ordem, $15 \mathrm{~Hz}$ ) e $1 \%$ para o método DSNI;

- os métodos PQ, RSP e RSN possuem filtros passa-baixas cuja resposta em freqüência influe no comportamento do sinal de compensação. Um tempo de resposta menor é obtido às custas de uma deterioração significativa no grau de compensação. $\mathrm{O}$ grau de desequilíbrio passou a ser menor que $20 \%, 24 \%$ e $40 \%$ respectivamente para os métodos PQ, RSP, RSN utilizando-se um filtro passa baixa de $2^{\circ}$ ordem, $80 \mathrm{~Hz}$;

- a extração somente da parcela de seqüência negativa visando a compensação somente dos desequilíbrios de carga apenas é possível pelos métodos DTRVE, RSN e DSNI;

- todos os métodos permitem a correção de harmônicos de corrente (a utilização do método DSNI, em conjunto com outros métodos, tendo como objetivo a compensação de desequilíbrios e harmônicos constituí objeto de pesquisa do autor, tem se mostrado promissora e em breve será publicada);

- todos os métodos necessitam de filtro passa-baixa com exceção do método proposto (DSNI), estando assim sujeitos às características deste filtro.

- os métodos RSP e RSN necessitam pela própria concepção do método de um circuito de sincronismo PLL (PhaseLocked Loop); se o objetivo for a obtenção de correntes senoidais é necessária a utilização de um circuito PLL pelo método PQ [35]; a princípio não há necessidade de um circuito PLL para os métodos DTRVE e DSNI, no entanto, variações na freqüência da rede ocasionam uma deterioração na obtenção das correntes de compensação de seqüência negativa. Para uma variação de $+-5 \%$ na freqüência da rede
$(60 \mathrm{~Hz})$, houve uma deterioração significativa no método DTRVE (grau de desequilíbrio passou a ser próximo de $15 \%$ ) e um menor no método DSNI (grau de desequilíbrio passou a ser próximo de 4\%). A diminuição do grau de desequilíbrio pode ser obtida para ambos os métodos através de um circuito PLL com objetivo de realizar o sincronismo entre os pulsos de amostragem e a rede.

Pelas observações feitas em [38], pode-se concluir que quando se trata da compensação de desequilíbrios da componente fundamental o método proposto de injeção direta de correntes de seqüência negativa (DSNI) é o mais eficiente, sendo de fácil implementação, apresentando um tempo de resposta bem menor e uma compensação mais efetiva que os outros métodos.

\section{IMPLEMENTAC̄̃̃O DO CONVERSOR DE INJEÇÃO DE CORRENTE DE COMPENSAÇÃO}

Uma malha de controle da corrente é necessária para que a corrente de compensação injetada pelo conversor na rede CA siga a referência de corrente calculada em tempo real. Existem várias estratégias de implementação da malha de corrente [5], [6], [16], [17], [22] e [24] destacando-se o controlador linear analógico, o controle por banda de histerese e o controle do tipo "deadbeat". Uma comparação entre as diferentes estratégias de controle pode ser encontrada em [5], [17] e [22].

O controle do tipo "deadbeat" segundo [5], [16] e [22] é uma técnica que prediz no começo de cada período de amostragem $(\Delta)$ o comportamento da corrente injetada. Baseia-se no erro atual entre a corrente injetada e a corrente de referência, nos parâmetros da carga e de outras variáveis. A tensão a ser gerada pelo PWM durante o próximo ciclo de modulação é determinada de modo a eliminar o erro no final do ciclo de amostragem. O controle deadbeat é baseado em um modelo do conversor, que é utilizado na estimativa do comportamento dinâmico do sistema. O controle é assim sensível aos parâmetros adotados no modelo. O objetivo do controle deadbeat é tornar nulo o erro para o próximo instante de amostragem $(\mathrm{k}+1)$, independente do erro no instante atual (k) como pode ser vista na Fig.2.

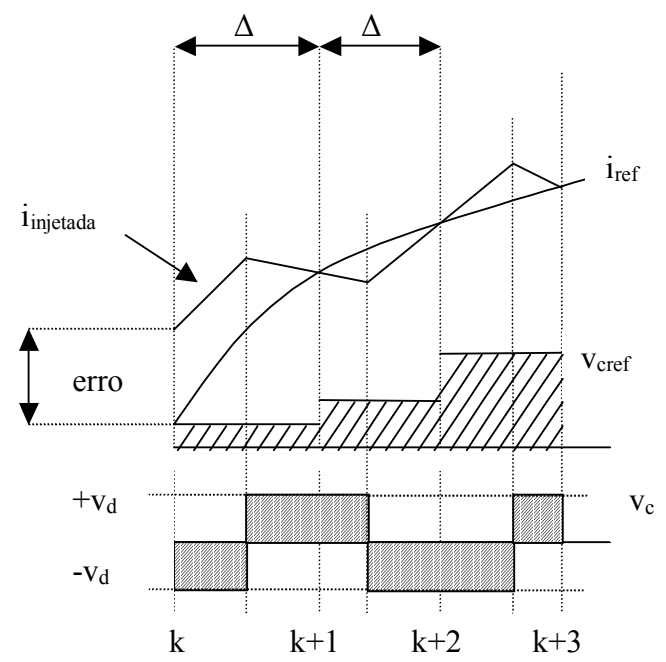

Instantes de amostragem

Fig. 2. Controle Deadbeat 
Como o conversor utilizado é do tipo fonte de tensão, deve-se calcular a referência de tensão a ser imposta no conversor conforme a eq.(2).

$$
\mathrm{v}_{\text {cref }}(\mathrm{k})=-\frac{\mathrm{L}}{\Delta} \cdot\left(\mathrm{i}_{\text {ref }}(\mathrm{k}+1)-\mathrm{i}_{\text {injetada }}(\mathrm{k})\right)+\mathrm{v}(\mathrm{k})
$$

Este controle apresenta as seguintes características:

- é inerentemente digital;

- permite a atuação independente por fase;

- tem atuação rápida;

- não requer filtro anti-aliasing;

- é sensível aos parâmetros adotados do modelo;

- apresenta freqüência fixa de chaveamento;

$\mathrm{Na}$ configuração de um conversor do tipo VSI é necessário um capacitor no barramento CC. Este capacitor é dimensionado de modo a suprir as potências oscilatórias que o conversor deve compensar. Para que o conversor possa operar satisfatoriamente injetando correntes de compensação na linha é necessário que a tensão no capacitor $\mathrm{v}_{\mathrm{dm}}(\mathrm{t})$ tenha um valor maior que o pico da tensão da rede. Durante os transitórios no sistema elétrico (tensão $\mathrm{CA}$, carga) o compensador pode absorver (injetar) elevados valores de potência ativa instantânea fazendo com que a tensão do barramento CC aumente (diminua). Assim, torna-se necessário que o capacitor consiga absorver (fornecer) o fluxo de energia sem que sua tensão ultrapasse os valores mínimo e máximo pré-estabelecidos. Terminado o transitório, deve-se restabelecer a tensão $\mathrm{v}_{\mathrm{dm}}(\mathrm{t})$ no valor estipulado $\left(\mathrm{v}_{\mathrm{d} \text { ref }}\right)$, através da malha de controle da tensão no capacitor [38].

O diagrama de blocos mostrando as malhas de controle da corrente $\mathrm{CA}$ e da tensão no barramento $\mathrm{CC}$ é visto na Fig.3.

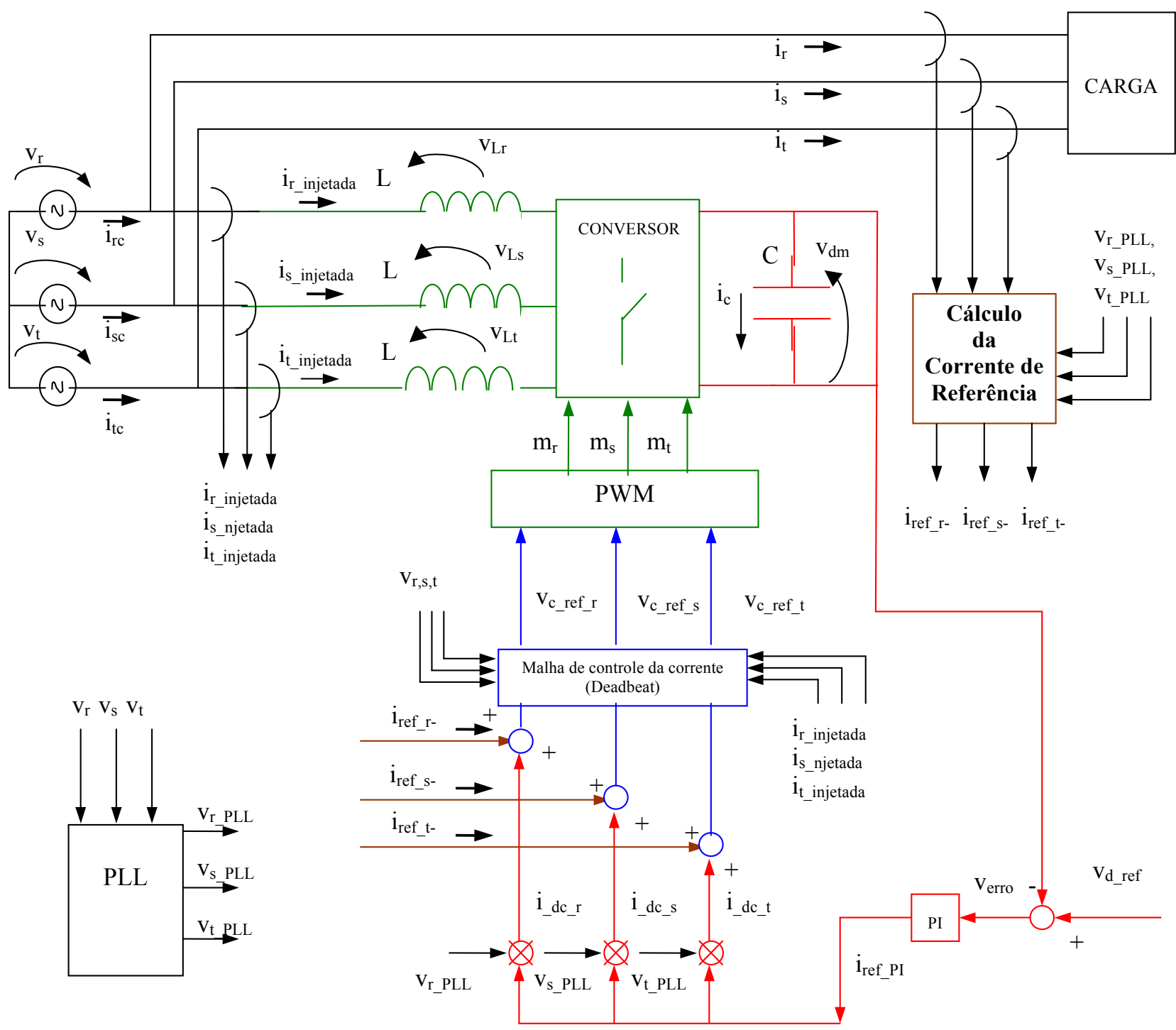

Fig. 3. Diagrama de bloco mostrando o conversor e as malha de controle de tensão no barramento CC e da corrente CA

A tensão sobre o capacitor $\left(\mathrm{v}_{\mathrm{dm}}\right)$ é subtraída da tensão de referência $\left(\mathrm{v}_{\mathrm{d} \_ \text {ref }}\right)$, gerando a tensão de erro $\left(\mathrm{v}_{\text {erro }}\right)$, que é aplicada ao controlador PI. Multiplica-se a corrente de saída do controlador PI ( $\left.\mathrm{i}_{\text {ref PI }}\right)$ pela tensão unitária de cada fase obtida de um circuito PLL ( $\mathrm{v}_{\mathrm{r}_{\mathrm{B}} \text { PLL }}, \mathrm{v}_{\mathrm{s}}$ PLL, $\mathrm{v}_{\mathrm{t}}$ PLL), produzindo os sinais $\left(i_{d c r}, i_{d c ~ s}, i_{d c t}\right)$ em fase com as tensões da rede $e$ 
responsáveis pela injeção (absorção) de potência ativa no capacitor se $i_{\text {ref_PI }}>0\left(i_{\text {ref_PI }}<0\right)$. Estes sinais são somados aos sinais correspondentes as correntes de referência de cada fase

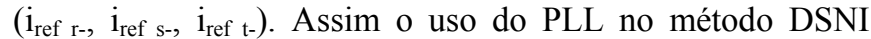
pode ter dupla função : a geração da "corrente para a malha de tensão" em fase com a tensão da rede e propiciar o sincronismo entre os pulsos de amostragem e a rede aumentando a performance do método quando sujeito a variações de freqüência da rede (item IV).

Para a simulação completa do sistema foi utilizado o software Matlab- Simulink-Power System Blockset com passo de amostragem de $1 \mu$ s.

A carga simulada é uma carga variável (Fig.4), seu comportamento pode ser dividido em três partes com as seguintes características:

a) sistema sem carga é submetido a um degrau de carga que caracteriza o sistema com o máximo desequilíbrio possível

- inicialmente o sistema se encontra sem nenhuma carga até $0,005 \mathrm{~s}$;

- no intervalo de $0,005 \mathrm{~s}$ até $0,06 \mathrm{~s}$ é ligada uma carga desbalanceada (uma carga monofásica de $1 \Omega$ é ligada entre as fases $\mathrm{R}$ e $\mathrm{S}$ ), caracterizando uma situação de máximo desequilíbrio possível (a amplitude da seqüência positiva na linha (1 A) é igual a amplitude da seqüência negativa na linha (1 A));

b) submete-se o sistema desbalanceado a um degrau de carga de forma a balanceá-lo
- no intervalo de $0,06 \mathrm{~s}$ a $0,12 \mathrm{~s}$ o sistema recebe um degrau de carga de modo a balanceá-la, tornando-se uma carga em triângulo, com três resistências de $1 \Omega$. Tem-se apenas uma corrente de seqüência positiva, com amplitude de $3 \mathrm{~A}$;

c) Adiciona-se ao sistema uma carga não linear com harmônicos

- após 0,12 s somam-se a cada fase da carga do sistema um sinal harmônico com freqüência de $180 \mathrm{~Hz}$, amplitude igual a $0,5 \mathrm{~A}$ e com fases $\left(0^{\circ}\right.$ (fase $\mathrm{r}$ ), $120^{\circ}$ (fase $\mathrm{s}$ ) e $-120^{\circ}$ (fase $\mathrm{t}$ )) ( $3^{\circ}$ harmônica de seqüência negativa), em paralelo com sinal harmônico com freqüência de $300 \mathrm{~Hz}$, amplitude igual a 0,2 A e com fases $\left(-30^{\circ}\right.$ (fase $\left.r\right),-150^{\circ}$ (fase s) e $90^{\circ}$ (fase $\left.t\right)$ ) ( $5^{\circ}$ harmônica de seqüência positiva), em paralelo com uma resistência $3 \Omega$;

Todas as simulações são apresentadas com condições quiescentes em " $\mathrm{t}=0$ ", exceto o capacitor do barramento $\mathrm{CC}$, que se encontra previamente carregado em 2,3 V.

$\mathrm{O}$ método proposto (DSNI) para o cálculo da componente de seqüência negativa da corrente e sua compensação, apresentado no item III é simulado para uma carga variável ligada em triângulo. Todas as amplitudes das tensões e correntes são representadas pelos seus valores de pico, exceto quando especificado em contrário. As tensões de faseneutro são simétricas e equilibradas com amplitude de $1 \mathrm{~V}$ e freqüência de $60 \mathrm{~Hz}$.

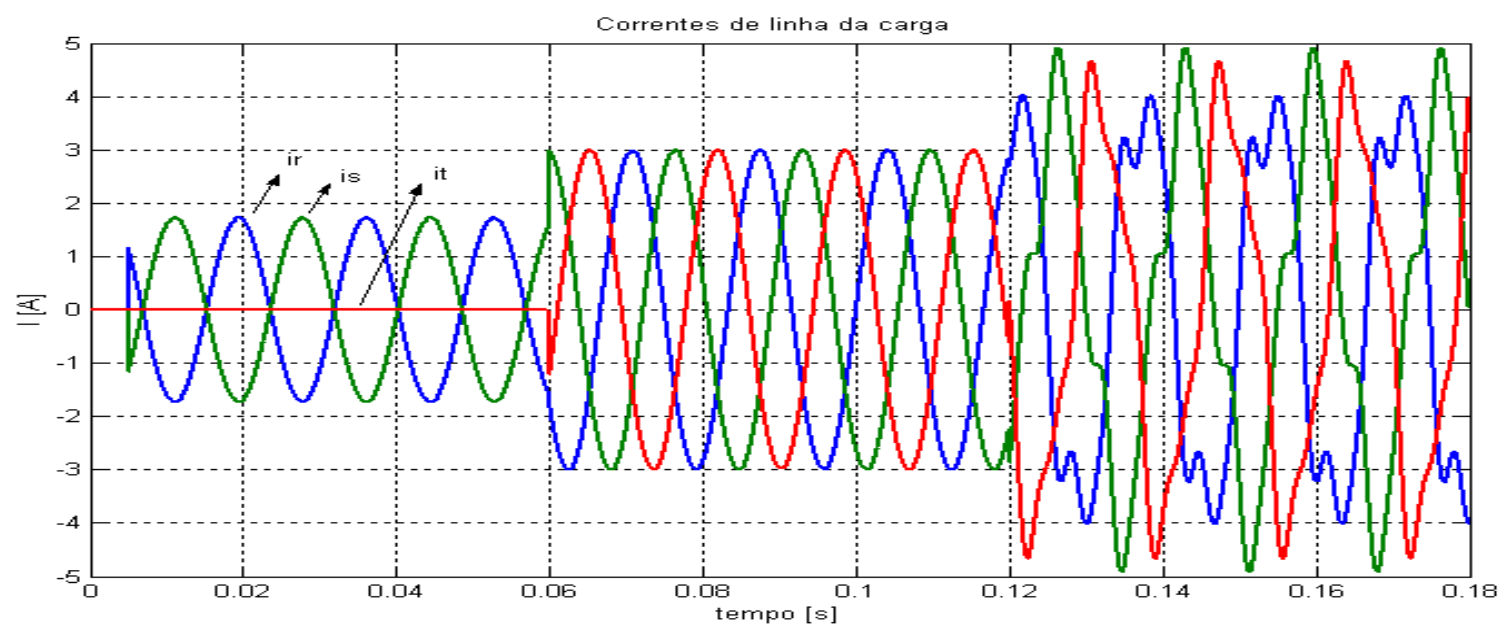

Fig. 4. Correntes de linha da carga variável

Apresentam-se a seguir, as formas de onda da corrente de referência para a compensação pelo filtro e das correntes na rede após a compensação, respectivamente (Figs.5 e 6). Nas Figs.7 e 8 são apresentadas respectivamente à variação da tensão no barramento $\mathrm{CC}$ e a atuação da malha de corrente para uma das fases (fase r). Comentários referentes ao comportamento do método DSNI na presença de harmônicos se encontram no anexo A.

Observa-se pela Fig.5 que a corrente de referência imposta ao filtro corresponde ao esperado, sendo composta apenas pela parcela referente à compensação da seqüência negativa da corrente da carga. Na Fig.6, observa-se que a compensação foi realizada no tempo previsto de $1 / 4$ de ciclo compensando o desequilíbrio do sistema.

Pode-se notar que a tensão no barramento $\mathrm{CC}$ vista na Fig.7 apresenta um afundamento no momento do degrau de carga e que a tensão no barramento volta a subir devido a atuação da malha de tensão forçando a injeção de potência ativa de média não nula no capacitor. Após cada aumento de carga, o atraso de $1 / 4$ de ciclo do bloco que calcula as correntes de referência, faz com que as correntes na rede variem de modo gradual. Como a carga variou de modo 
abrupto, o compensador deverá alimentar a carga durante o transitório, descarregando o capacitor.

O comportamento da malha de corrente na fase $r$ é apresentado na Fig.8. Nota-se o bom funcionamento da malha de corrente.

\section{VII.CONCLUSÕES}

Como dito anteriormente, o valor da potência aparente associada aos reativos e desequilíbrios é maior do que a associada aos harmônicos. Assim, a compensação de distúrbios de baixa freqüência (reativos e desequilíbrios) é feita por meio de um conversor chaveado de elevada potência operando com menor freqüência de chaveamento enquanto que os harmônicos podem ser tratados com um conversor de menor potência e alta freqüência de chaveamento. As diversas comparações entre os métodos para o cálculo em tempo real da corrente de referência do conversor PWM servem como base para a escolha do método adequado de acordo com a necessidade de compensação.

Quando o objetivo é somente a compensação de desequilíbrios das correntes da rede o método proposto de injeção direta de correntes de seqüência negativa (DSNI) se mostrou o mais eficiente, apresentando resposta rápida com menor complexidade de cálculo. O tempo de resposta de $1 / 4$ de ciclo e sua fácil implementação, tornam sua utilização uma opção viável.

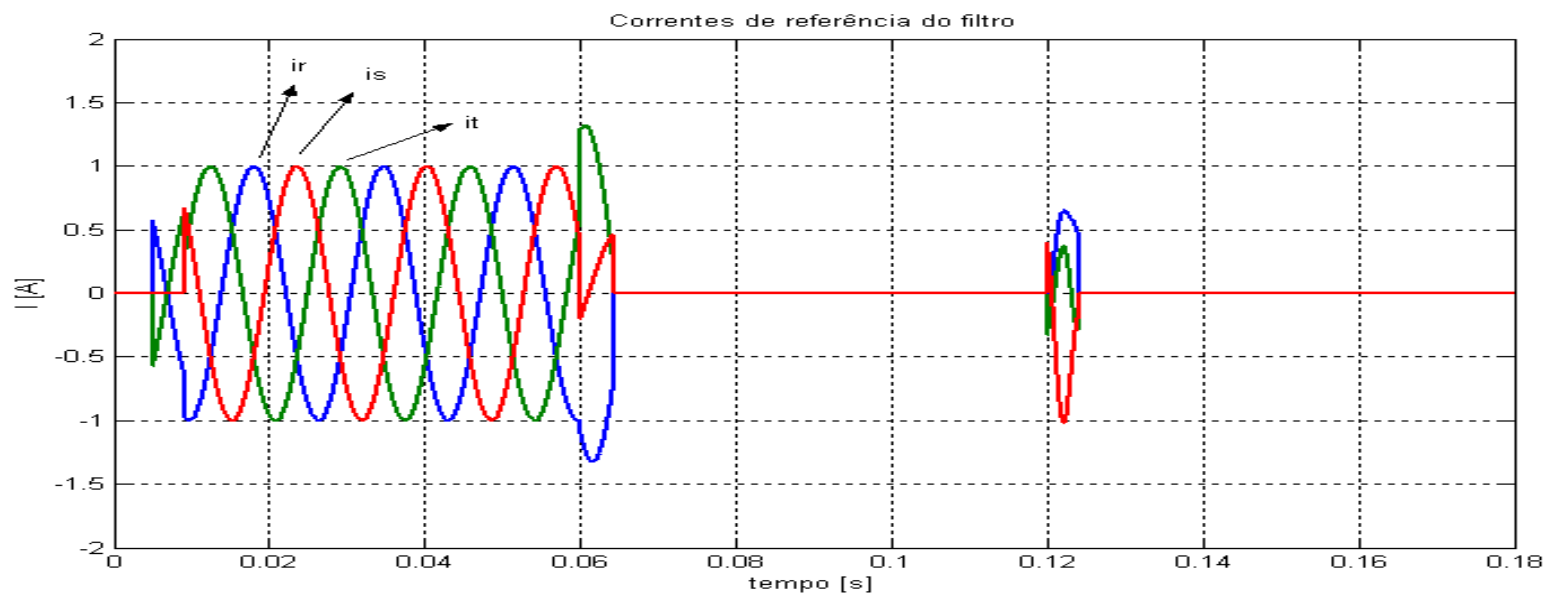

Fig. 5. Correntes de referência para o sistema de compensação

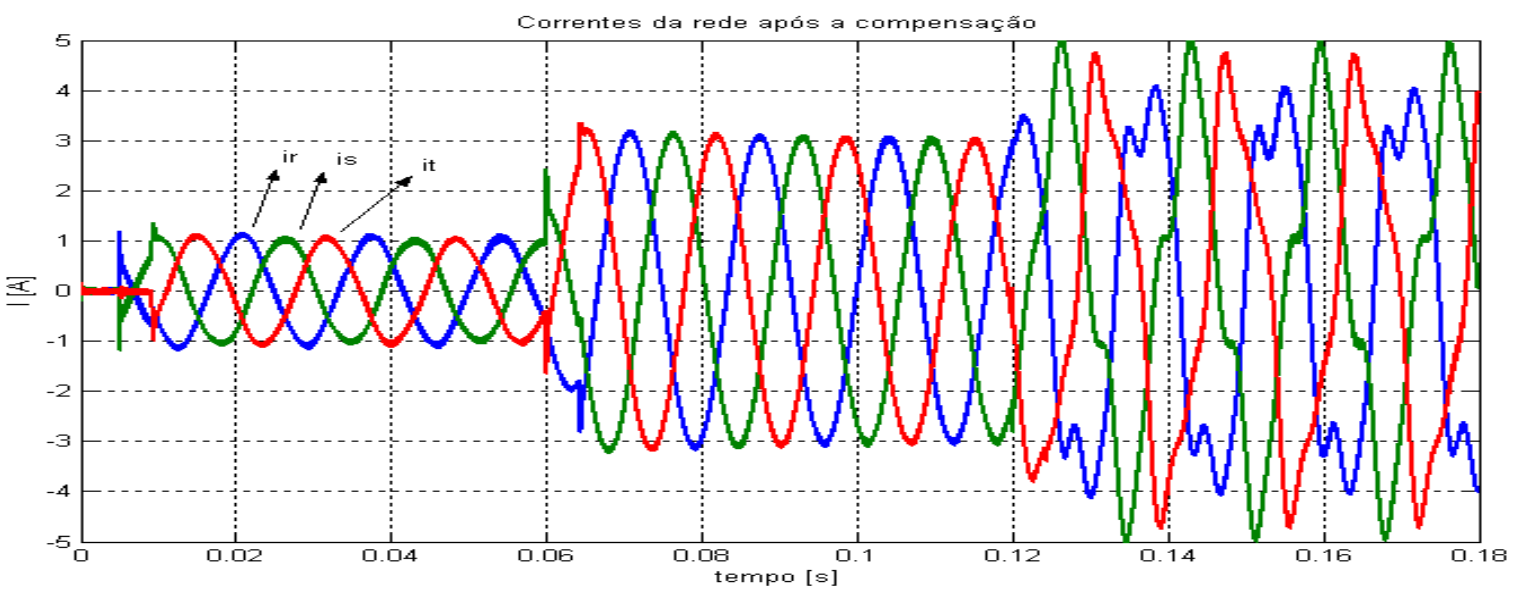

Fig. 6. Correntes de linha na rede após a compensação

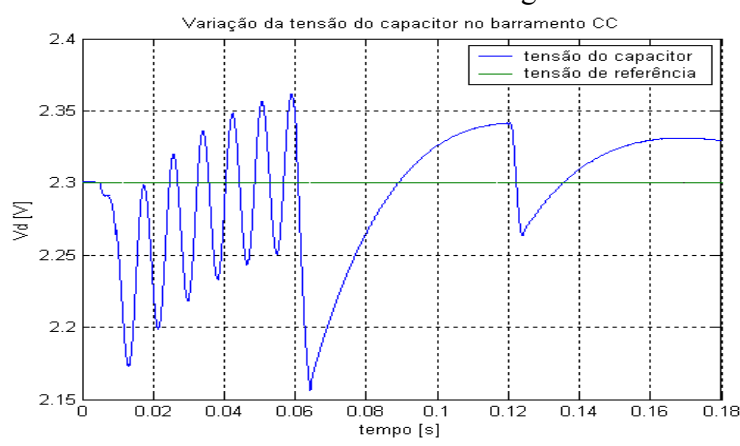

Fig. 7. Variação da tensão no barramento CC

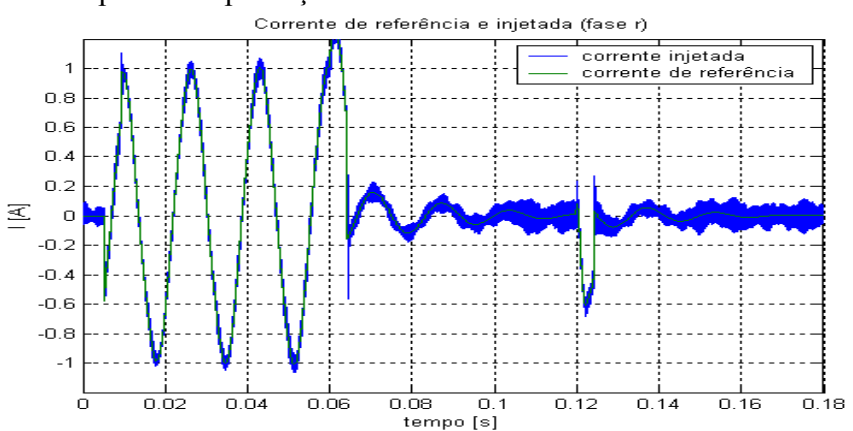

Fig. 8. Comportamento da malha de corrente (fase r) 


\section{ANEXO A - MÉTODO PROPOSTO DE EXTRAÇÃO DE SEQÜÊNCIA NEGATIVA - INJEÇÃO DIRETA DE SEQÜÊNCIA NEGATIVA (DSNI) - DEDUÇÃO DO ALGORITMO}

Sabe-se de [29] e [39] que a matriz que relaciona as componentes simétricas fasoriais da corrente de linha com as componentes fasoriais da corrente de linha é dada pela eq.(A1).

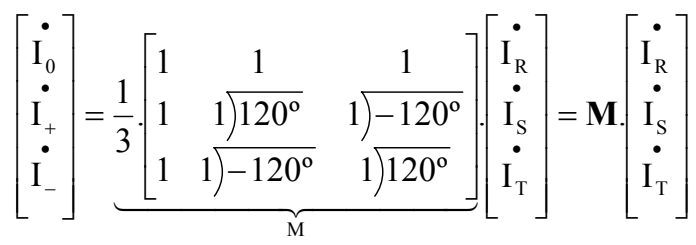

Para se eliminar o desequilíbrio existente entre as correntes de linha basta que se anulem as correntes de seqüência negativa e zero existentes na mesma. Assim, a corrente de compensação deve ter o sinal oposto à parcela de corrente negativa ( $-\dot{\mathrm{I}}_{-}$) (eq.(A-2), desprezando-se $\dot{\mathrm{I}}_{\mathrm{o}}$ ).

$$
\left[\begin{array}{c}
0 \\
0 \\
-\mathrm{I}_{-}
\end{array}\right]=-\frac{1}{3} \cdot\left[\begin{array}{ccc}
0 & 0 & 0 \\
0 & 0 & 0 \\
1 & 1)-120^{\circ} & 1) 120^{\circ}
\end{array}\right] \cdot\left[\begin{array}{c}
\bullet \\
\mathrm{I}_{\mathrm{R}} \\
\mathrm{I}_{\mathrm{S}} \\
\dot{\mathrm{I}_{\mathrm{T}}}
\end{array}\right]
$$

Para se obter as correntes de compensação na base rst basta multiplicar a corrente obtida pela eq.(A-2) pela matriz inversa $\left(\mathrm{M}^{-1}\right)$ da eq.(A-1), obtendo a eq.(A-3).

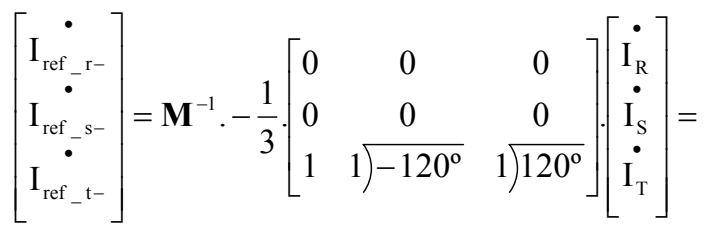

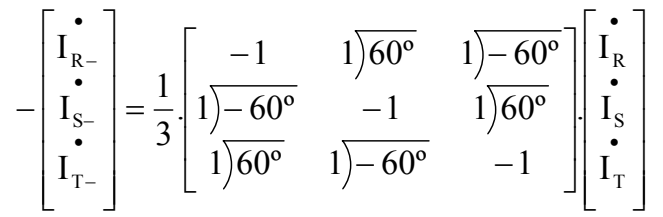

A matriz complexa da eq.(A-3) pode ser reescrita em duas sub-matrizes: uma contendo os termos reais e outra contendo os termos imaginários multiplicados por $-\mathrm{j}$ conforme a eq.(A4).

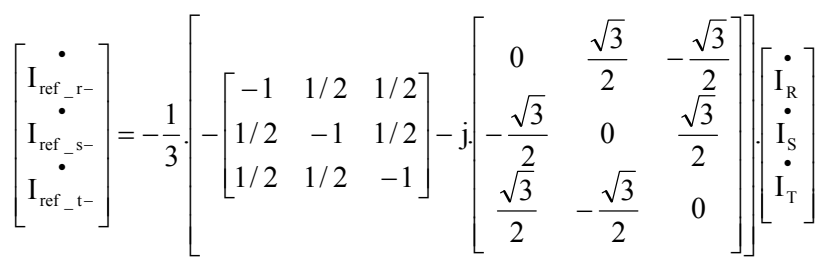

No domínio do tempo, o atraso de $90^{\circ}$ é implementado armazenando-se as N/4 últimas amostras medidas, onde $\mathrm{N}$ é igual ao número de amostras por ciclo da rede. As correntes atrasadas são denominadas por $\mathrm{i}_{\mathrm{r} 90}(\mathrm{t}), \mathrm{i}_{\mathrm{s} 90}(\mathrm{t})$ e $\mathrm{i}_{\mathrm{t} 90}(\mathrm{t})$. Reescrevendo a eq.(A-4) no domínio do tempo, para o regime permanente obtém-se a eq.(A-5).

$-\left[\begin{array}{l}\mathrm{i}_{\mathrm{r}-}(\mathrm{t}) \\ \mathrm{i}_{\mathrm{s}-}(\mathrm{t}) \\ \mathrm{i}_{\mathrm{t}-}(\mathrm{t})\end{array}\right]=-\frac{1}{3} \cdot\left[-\left[\begin{array}{ccc}-1 & 1 / 2 & 1 / 2 \\ 1 / 2 & -1 & 1 / 2 \\ 1 / 2 & 1 / 2 & -1\end{array}\right]\left[\begin{array}{l}\mathrm{i}_{\mathrm{r}}(\mathrm{t}) \\ \mathrm{i}_{\mathrm{s}}(\mathrm{t}) \\ \mathrm{i}_{\mathrm{t}}(\mathrm{t})\end{array}\right]+\left[\begin{array}{ccc}0 & \frac{\sqrt{3}}{2} & -\frac{\sqrt{3}}{2} \\ -\frac{\sqrt{3}}{2} & 0 & \frac{\sqrt{3}}{2} \\ \frac{\sqrt{3}}{2} & -\frac{\sqrt{3}}{2} & 0\end{array}\right] \cdot\left[\begin{array}{l}\mathrm{i}_{\mathrm{r} 90}(\mathrm{t}) \\ \mathrm{i}_{\mathrm{s} 90}(\mathrm{t}) \\ \mathrm{i}_{\mathrm{t} 90}(\mathrm{t})\end{array}\right]\right]$

Observa-se também que se a carga a ser compensada for uma carga monofásica contendo harmônicos, estes harmônicos serão redistribuídos entre as fases apresentando uma amplitude menor que a inicial e defasagem de $120^{\circ}$. Como o sinal analisado é defasado baseado na freqüência fundamental, dependendo da ordem da harmônica analisada poderá haver uma inversão de fase resultando na compensação da seqüência positiva e não negativa do harmônico, isto ocorre para harmônicos de ordem 3, 7, 11, 15 e assim por diante, o inverso ocorrendo com harmônicos de 5, 9, 13 ordem e assim por diante. Esta característica adicional de compensação parcial de harmônicos presente no método proposto é discutida em um artigo apresentado no Congresso Brasileiro de Automática CBA'2004.

\section{REFERÊNCIAS BIBLIOGRÁFICAS}

[1]ABELlÁN, A.; GARCERÁ, G.; BENAVENT, J. A New Control Method for Obtaining Reference Currents of Shunt Active Power Filters in Unbalanced and Non-Sinusoidal Conditions. In:Industrial Electronics- ISIE'99 Proceedings of the IEEE International Symposium on,Jul.1999. Anais eletrônicos. v.2.

[2]AKAGI, H.; NABAE, A. The p-q Theory in Three-Phase Systems Under Non-Sinusoidal Conditions. ETEP, v.3, n.1, Jan. Feb. 1993.

[3]BHAVARAJU, V. P.; ENJETI, P. N. Analysis and Design of an Active Power Filter for Balancing Unbalanced Loads. IEEE Transactions on Power Electronics, v.8, n.4, Oct. 1993.

[4]BRITTAIN, J. C. Charles F. Scott : A Pioneer in Electrical Power Engineering. IEEE Industry Applications Magazine, p.6-8. Nov. Dec. 2002.

[5]BUSO, S.; MALESANI, L.; MATTAVELLI, P. Comparison of Current Control Techniques for Active Filter Applications. IEEE Transactions on Industrial Electronics, v.45, n.5, Oct. 1998.

[6]BUSO, S. Digital Control of Power Converters (Lecture Notes). Disponível

$<\mathrm{http} / / /$ www.dsce.fee.unicamp.br/ antenor/Digital.html>. Acesso em:2003.

[7]CHANG, G. W.; SHEE, T. A Comparative Study of Active Power Filter Reference Compensation Approaches. IEEE 2002 Power Engineering Society Summer Meeting, 21-25 July 2002. Anais, IEEE, v.2, p.10171021. 2002.

[8]CHEN,C. C.; HSU, Y. A Novel Approach to the Design of a Shunt Active Filter for na Unbalanced Three-Phase Four Wire System under Nonsinusoidal Conditions. In:Power Engineering Society Summer Meeting.Jul.2000. Anais eletrônicos. v.2. p.1059.

[9] CHEN, Tsai-Hsiang. Criteria to estimate the Voltage Unbalances due to High-Speed Railway Demands. IEEE Transactions on Power Systems, v.9, n.3, Aug. 1994

[10]CHOI, S.; LEE, W.; HYUN, D.; LEE, T. The Control system of the Active Power Filter Considering Power Factor in Unbalanced Load. 
In:Industry Applications Conference, Oct.2000. Anais eletrônicos. v.4 p.2123-2128.

[11]CUTRI, R.; MATAKAS JR., L. Reference Currents Determination Techniques For Load Unbalance Compensation. In: $7^{\circ}$ Congresso Brasileiro de Eletrônica de Potência- COBEP'03,Fortaleza,Set.2003. CD-ROM

[12]CZARNECKI, L. S. Power Factor Improvement of Three-Phase Unbalanced Loads with Non-Sinusoidal Supply Voltage. ETEP, v.3, $\mathrm{n}^{\circ} 1$, Jan. Feb. 1993.

[13]E.E.STAFF DEL M.I.T. Circuitos Magnéticos Y Transformadores Madri:Editorial Reverte S.A., 1965. p.638-640.

[14]DIXON, J. W.; GARCIA, J.; MORÁN, T. L. A Control System for a Three Phase Active Power Filter which Simultaneously Compensates Power Factor and Unbalanced Loads. IEEE Transactions on Industrial Electronics, v.42, Issue:6, p.636-641. Dec. 1995

[15]HOCHGRAF, C.; LASSETER, R. Statcom Controls for Operation with Unbalanced Voltages. IEEE Transactions on Power Delivery, v.13. n.2, Apr. 1998.

[16]JUNQUEIRA, A. D.; MATAKAS JR., L.; KOMATSU, W. Digital Implementation of Three-Phase Rectifier with Deadbeat Controller. Revista Eletrônica de Potência, v.7., n.1, Nov. 2002.

[17]KAZMIERKOWSKI, M. P.; DZIENIAKOWSKI, M. A. Review of Current Regulation Methods For VS-PWM Inverters Industria Electronics. Conference Proceedings, ISIE'93 - Budapest, IEEE International Symposium on, 1-3 June 1993, p.448-456. 1993

[18]KIM, H.; BLAABJERG, F.; JENSEN, B. B.; CHOI, J. Instantaneous Power Compensation in Three-Phase Systems by Using p-q-r theory. In:Power Electronics Specialists Conference,2001-PESC'2001- IEEE 32nd Annual, June.2001. Anais eletrônicos. v.2. p.478-485.

[19]KIM, H. Control an Active Filter in Unbalanced Non- sinusoida Source Voltages. In:Power Conversion Conference Proceedings of. Osaka, Apr.2002. Anais eletrônicos. v.2. p.831-836. Acesso restrito disponível em:<periódicos IEEE-Capes>.

[20]LEDWICH, G.; GEORGE, T. A. Using Phasors to analize power system negative phase sequence voltages caused by unbalanced loads IEEE Transactions in Power Systems, v.9, n.3, Aug. 1994.

[21]LIN, C. E.; CHEN, C. L.; HUANG, C. L. Calculating Approach and Implementation for active Filters in Unbalanced Three-Phase System using Synchronous Detection Method. In:Industrial Electronics,Control,Instrumentation and Automation - 'Power Electronics and Motion Control', Proceedings of the 1992 International Conference on,Nov.1992. Anais eletrônicos. v.1. p.374-380.

[22]MALESANI, L.; TOMASIN, P. PWM Current Control Techniques of Voltage Source Converters - A Survey. Industrial Electronics, Control, and Instrumentation, 1993. Proceedings of the IECON '93., International Conference on, 15-19 Nov. 1993. Anais. v.2. p.670-675. 1993.

[23]MARQUES, G. D. A Comparison of Active Filters Control Methods in Unbalanced and Non-sinusoidal Conditions. In:Industrial Electronics Society - IECON'98 Proceedings of the 24th Annual Conference of the IEEE,Aug.Sep.1998. Anais eletrônicos. v.1. p.444-449. Acesso restrito disponível em:<periódicos IEEE-Capes $>$.

[24]MASWOOD, A. I. A PWM Voltage Source Inverter with PI Controller for Instantaneous Motor Current Control. Power Electronics and Drive Systems, 1995., Proceedings of 1995 International Conference on, 21-24 Feb. 1995. v.2. p.834-837. 1995

[25]MATSUI, M.; FUKAO, T. A Detecting Method for Active-ReactiveNegative Sequence Powers ans its Application. IEEE Transactions on Industry Applications, v.26, n.1, Jan. Feb. 1990.

[26]MCGRANAGHAM, M. Active Filter Design and Specification for Control of Harmonics in Industrial and Commercial Facilities. Electrotek Concepts,Inc. Knoxville TN, USA. Disponível em: $<$ http://www.dranetz-bmi.com/pdf/activeFilter.pdf $>$. Acesso em:2003.

[27]NIKOLAENKO, V. G. Optimal Balancing of Large Unbalanced Loads Using Shunt Compensators. In:Harmonics and Quality of Power Procceedings 8th International Conference on,Oct.1998. Anais eletrônicos. v.1. p.537-542.

[28]PENTEADO JR., A. A. Compensadores estáticos para desequilíbrios : um procedimento de especificação e de análises das interferências no sistema elétrico. $1985 . \quad$ Tese (Doutorado) - Escola Politécnica,Universidade de São Paulo. São Paulo.

[29]ROBBA, J. et al. Introdução a Sistemas Elétricos de Potência. 2ed. São Paulo:Edgard Blucher, 1996.

[30]SAMESIMA, M. I. Compensadores Estáticos de Reativos e de Desequilíbrios em Sistemas Elétricos de Potência. 1984. Dissertação (Mestrado) - Escola Politécnica,Universidade de São Paulo. São Paulo.

[31]SENINI, S.; WOLFS, P. Hybrid Active Filter for Harmonically Unbalanced Three Phase Three Wire Railway Traction Loads. IEEE Transactions on Power Electronics, v.15, n.4, July. 2000.

[32]SENINI, S. T.; WOLFS, P. J. Novel Topology of Unbalanced Load in Single Phase Electric Traction Systems. In:Power Electronics Specialists Conference,2001-PESC'2002- IEEE 33rd Annual,June.2002. Anais. .3. p.1208-1212. 2002

[33]VERDELHO, P.; MARQUES, G. D. Design and Performance of an Active Power Filter and Unbalanced Current Compensator. In:Industrial Electronics,Control and Instrumentation,Sep.1994. IECON'94,20th International Conference on. Anais eletrônicos. 1994. v.1. p.422-427.

[34]WATANABE, E.; AREDES, M. Teoria de Potência Ativa e Reativa Instantânea e Aplicações - Filtros Ativos e FACTS". Tutorial in the CBA'98 (XII Brazilian Automatic Control Conference), Uberlândia, Brazil, Sep.14-18, 1998

[35]WATANABE, E.; AREDES, M.; AKAGI, H. The p-q theory for active filter control : some problems and solutions. In:XIV Congresso Brasileiro de Automática-CBA,2002.

[36]WILlEMS, J. L. Current Compensation in Three-Phase Power Systems. ETEP, v.3, n.1, Jan. Feb. 1993.

[37]ZHANG, G.; XU, Z. A New Real-time Negative and Positive Sequence Componentes Detecting Method Based on Space Vector. In:POWER ENGINEERING SOCIETY WINTER MEETING 2001,Jan.-Feb.2001. Anais eletrônicos. IEEE, v.1. p.275-280.

[38]CUTRI, R. Compensação de desequilíbrios de carga empregando conversor estático operando com modulação em largura de pulso. 2004 Boletim Técnico - Escola Politécnica,Universidade de São Paulo. São Paulo. (no prelo)

[39]FORTESCUE, C. L. Method of symmetrical co-ordinates applied to the solution of polyphase networks. $34^{\text {th }}$ Annual Convention of the America Institute of Electrical Engineers. Atlantic City. N.J. June,28. 1918.

\section{DADOS BIOGRÁFICOS}

Rodrigo Cutri - nascido em São Caetano do Sul, São Paulo, é engenheiro eletricista pela Escola de Engenharia Mauá (2002) e mestre em Engenharia Elétrica pela Escola Politécnica da Universidade de São Paulo (2004). Atualmente, leciona nas áreas Matemática e Elétrica da Escola de Engenharia Mauá e está cursando o doutorado na área de Eletrônica de Potência na Escola Politécnica da Universidade de São Paulo. Seus interesses são: qualidade da energia elétrica e filtros ativos de potência.

Lourenco Matakas Jr. - é engenheiro eletricista (1983), mestre (1989) e doutor (1998) em Engenharia Elétrica pela Escola Politécnica da Universidade de São Paulo. Trabalhou como Professor Assistente na Universidade de Tóquio. Atualmente, trabalha na Universidade de São Paulo, na Universidade Católica de São Paulo e na Universidade São Judas. Suas áreas de pesquisa são: retificadores com alto fator de potência, modelamento e controle de conversores de potência, implementação de conversores de alta potência, e aplicações de conversores de potência aos sistemas elétricos. 